\title{
Inflammatory myofibroblastic tumor of renal pelvis presenting with iterative hematuria and abdominal pain: A case report
}

\author{
SHUIQING WU, RAN XU, HUASHENG ZHAO, XUAN ZHU, LEI ZHANG and XIAOKUN ZHAO \\ Department of Urology, The Second Xiangya Hospital of Central South University, Changsha, Hunan 410011, P.R. China
}

Received November 23, 2014; Accepted September 14, 2015

DOI: $10.3892 / 01.2015 .3767$

\begin{abstract}
Inflammatory myofibroblastic tumor (IMT) is a rare type of mesenchymal tumor, which may affect various organs. The preferential site for IMT in the genitourinary system is the urinary bladder, while the presence of IMT in the kidney, and particularly in the renal pelvis, is rare. In the present report, the case of a 43-year-old man who was admitted to the Department of Urology of The Second Xiangya Hospital of Central South University (Changsha, China) in July 2012, with complaints of iterative gross hematuria and abdominal pain unresponsive to antibiotics is described. Computed tomography and magnetic resonance imaging indicated a slightly enhanced mass in the left renal pelvis of $1.5 \mathrm{~cm}$ in diameter. On request of the patient, a left nephrectomy was then performed, based on a suspected diagnosis of renal pelvic carcinoma. However, analysis of the intraoperative fast-frozen section exhibited proliferation of compact spindle cells, suggesting IMT. Therefore, further ureterectomy was avoided, and the patient remained in healthy condition thereafter.
\end{abstract}

\section{Introduction}

Inflammatory myofibroblastic tumor (IMT) is a rare entity that was firstly reported in the lung, although it has also been observed in various extrapulmonary anatomic sites (1). IMT in the kidney is rare, particularly in the renal pelvis. To the best of our knowledge, only six cases of IMT of renal pelvis have been reported in the English literature thus far. The majority of these cases were misdiagnosed as renal pelvic carcinoma prior to surgery, due to the common clinical and pathological characteristics shared by these tumors, and nephroureterectomy was usually performed, based on the preoperative diagnosis of renal pelvic carcinoma (2). In the present case report, an IMT of renal pelvis is described, which presented with iterative hematuria

Correspondence to: Professor Xiaokun Zhao, Department of Urology, The Second Xiangya Hospital of Central South University, 139 Renmin Road, Changsha, Hunan 410011, P.R. China

E-mail: yuqing591@163.com

Key words: inflammatory myofibroblastic tumor, renal pelvis, diagnosis, treatment, intraoperative frozen-section and abdominal pain, thus mimicking renal pelvic carcinoma. On request of the patient, a left nephrectomy was subsequently performed, but further ureterectomy was not required, since post-surgical analysis of the specimen revealed proliferation of compact spindle cells, which confirmed the diagnosis of IMT.

\section{Case report}

A 43-year-old man, presenting with a three-month history of gross hematuria and abdominal pain, was admitted to the Department of Urology of The Second Xiangya Hospital of Central South University (Changsha, China) in July 2012. Prior to the date of admission, the patient had been subjected to anti-inflammatory treatment in another hospital, without experiencing any improvement in his symptoms. There were no remarks on the patient's history other than the fact that the patient had been smoking 20 cigarettes/day for the previous 20 years. Urinalysis revealed an elevated number of red blood cells displaying homogeneity. Routine blood examination indicated infection and anemia, with a leukocyte count of $12.1 \times 10^{9}$ cells/ 1 and hemoglobin levels of $10^{9} \mathrm{~g} / 1$. Other laboratory tests were negative, and urinary cytology did not detect malignant cells.

Computed tomography (CT) scan revealed the presence of a slightly enhanced mass of $1.5 \times 1.0-\mathrm{cm}$ in size in the left renal pelvis (Fig. 1). Magnetic resonance imaging (MRI) was performed to further improve the accuracy of the diagnosis. On MRI, the tumor presented slightly low intensity on T1-weighted images and blended intensity on T2-weighted images, and appeared moderately enhanced in the arterial phase (Fig. 2). Taken together, these clinical and radiological manifestations suggested renal pelvic carcinoma, and a left radical nephrectomy was consequently performed on request of the patient. Post-surgical macroscopical analysis of the specimen revealed a hard grey/brown tumor with obscure boundary located in the pelvis. An intraoperative fast-frozen section was then performed, since transitional cell carcinoma was suspected. The intraoperative pathological results indicated proliferation of compact spindle cells. Therefore, further ureterectomy was avoided, and the patient exhibited a good prognosis at 26 months of follow-up.

Analysis of the postoperative paraffin section revealed various cellular foci composed of compact spindle cells (Fig. 3). Mild atypia and mitotic activity were observed in focal areas. Diffuse lymphoplasmacytic inflammatory cells, including lymphocytes and neutrophilic leukocytes, infiltrated in the spindle cells. Immunohistochemical analysis 
A

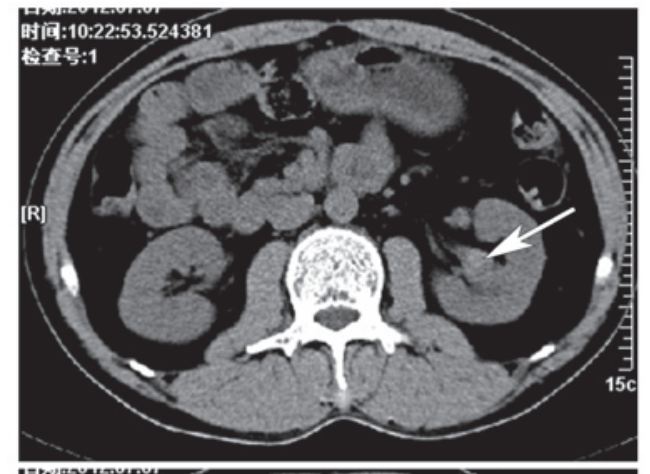

B

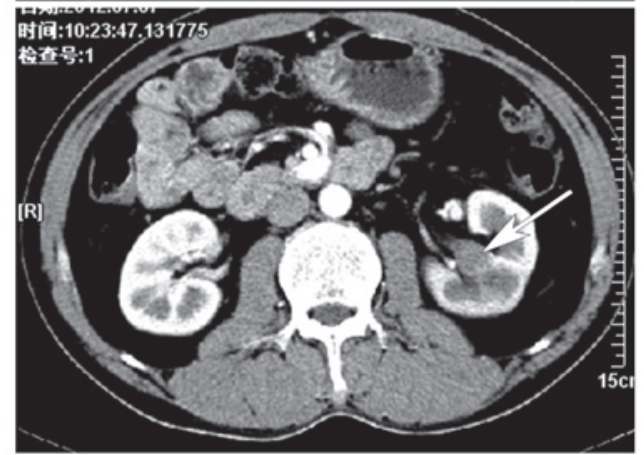

C

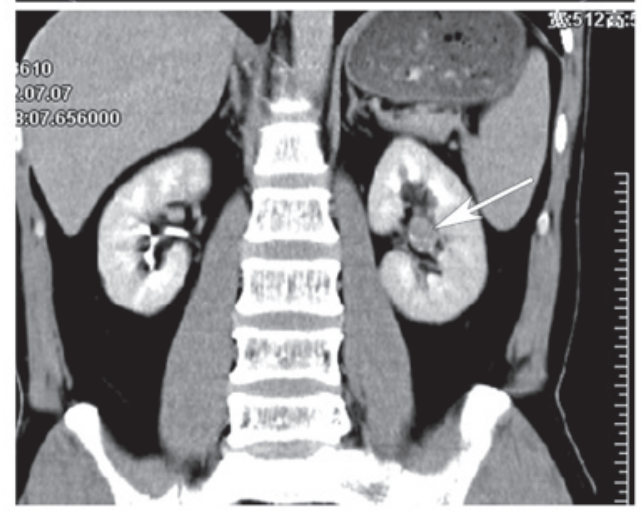

Figure 1. (A) CT scan revealed a $1.5 \times 1.0-\mathrm{cm}$ lesion located in the left renal pelvis, as indicated by the arrow in the image. (B) Axial and C) sagittal contrast-enhanced CT scans demonstrated a slightly enhanced tumor. CT, computed tomography.

with specific monoclonal antibodies against smooth muscle and muscle actin (and HHF35, respectively) demonstrated the specimen to be strongly positive for these markers. By contrast, the sample was negative for cluster of differentiation (CD)34, CD10, B-cell lymphoma 2, p53, anaplastic lymphoma kinase (ALK), Ki-67 and cytokeratin.

\section{Discussion}

IMT, also known as inflammatory pseudotumor or plasma cell granuloma, is a rare type of neoplastic lesion involving spindle cells. IMT was firstly reported as plasma cell granuloma in the lung, which is the main site of presentation for this tumor. In the genitourinary system, the urinary bladder has been described as the most common and frequently reported site of IMT (3). However, IMT of renal pelvis is a rare lesion, which mimics renal pelvic carcinoma, due to their similar characteristics.

IMT has been mostly reported in young patients, irrespective of their gender (4). The pathogenesis of IMT remains
A

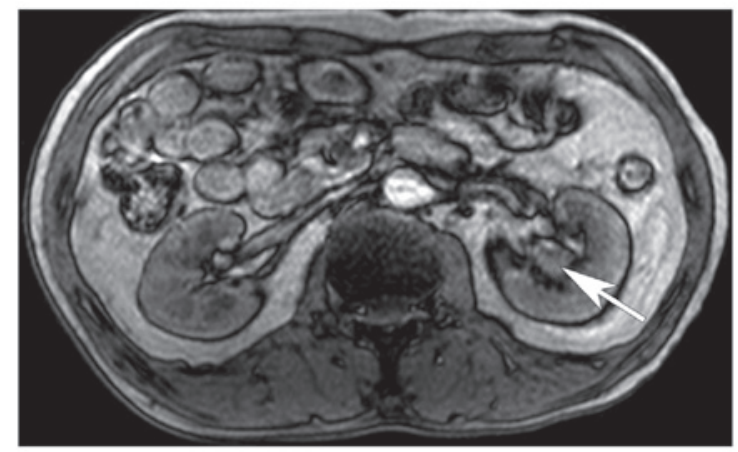

B

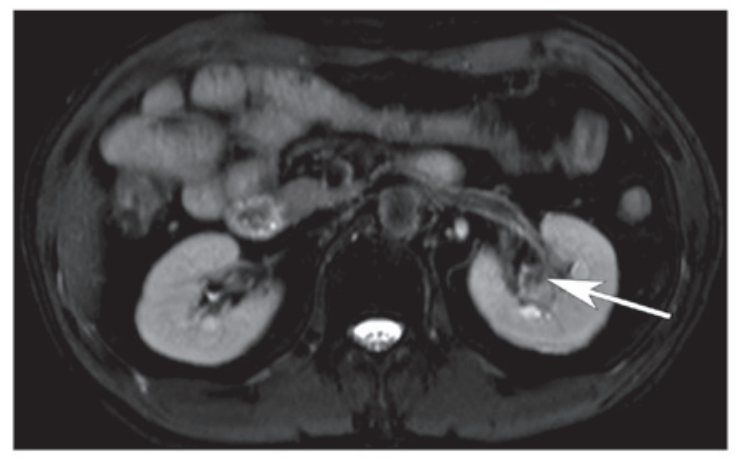

C

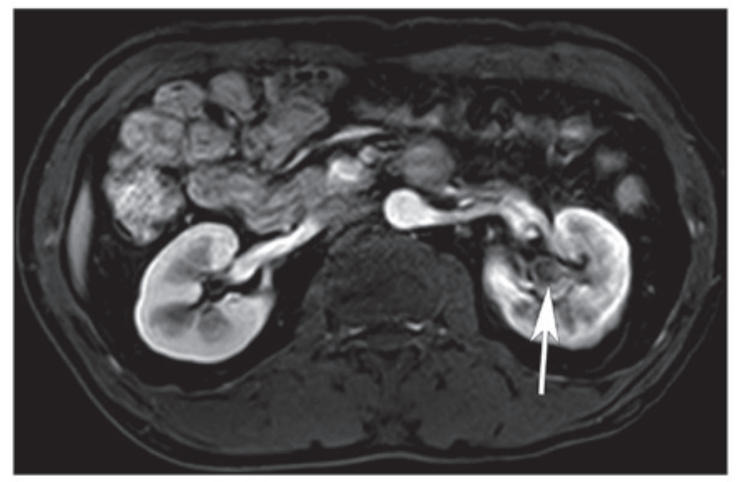

Figure 2. MRI revealed the presence of a heterogeneous mass of (A) slightly low intensity on T1-weighted images and (B) blended intensity on T2-weighted images, as indicated by the arrows in the image. (C) Enhanced-MRI demonstrated an unevenly enhanced tumor. MRI, magnetic resonance imaging.

unknown. Various factors have been considered as potential originators of IMT, including infection, trauma and autoimmunity (5). Infection has been considered as a significant factor in the initiation of IMT, and several bacteria and viruses have been associated with the development of IMT, including Epstein-Barr virus, human herpes virus, Bacillus sphaericus and Mycobacterium tuberculosis (6). Recently, Li et al (7) described the case of a patient with IMT of kidney, who presented a long-term history of hepatitis B. Therefore, it can be hypothesized that hepatitis B may lead to the development of IMT. In the patient of the present case report, the increase in leukocyte count may have been involved in the development of the IMT of renal pelvis observed in this patient.

In previous studies, hematuria was considered the main symptom exhibited by patients with IMT of renal pelvis, although prolonged fever and abdominal pain were also observed in certain patients (8). The imaging features of IMTs of renal pelvis are nonspecific, and may vary in CT and MRI, presenting homo- or heterogeneity. In addition, hypo-, iso-, or hyperdensity may be observed in enhanced CT, thus mimicking 
A

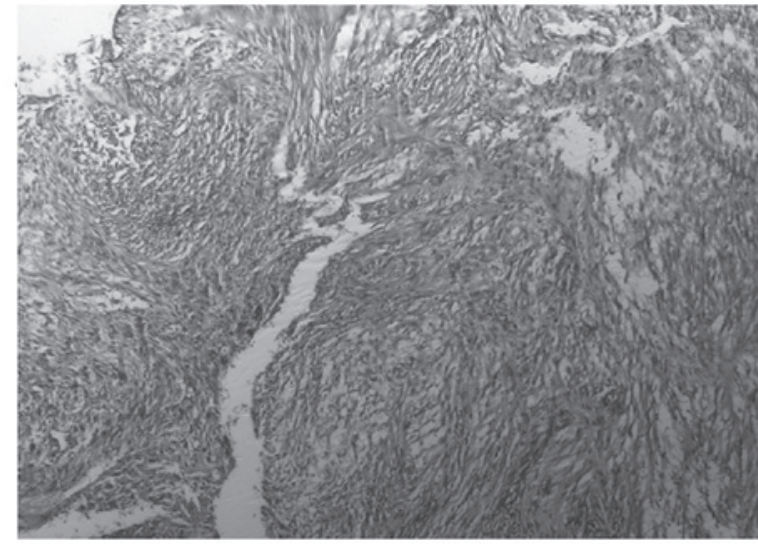

B

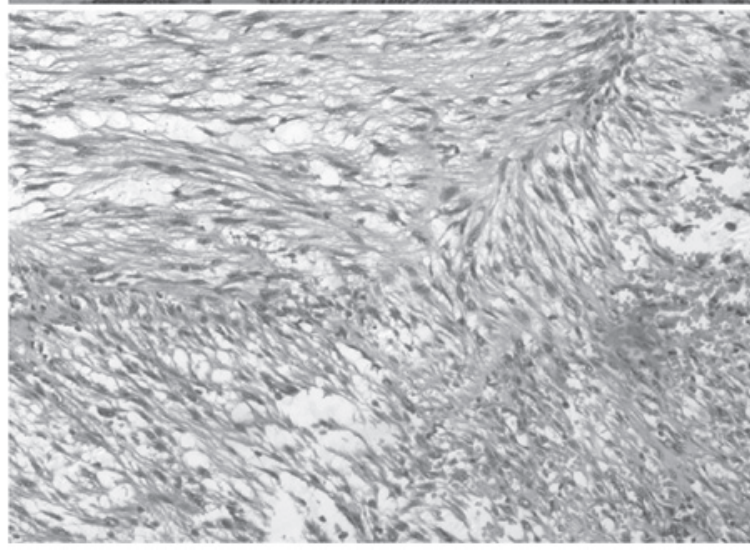

Figure 3. Microscopic findings following hematoxylin and eosin staining (A) Proliferation of compact spindle cells (magnification, x40). (B) Spindle fibroblast-like cells and collagen with infiltrating inflammatory cells and erythrocytes (magnification, x100).

transitional cell carcinoma and mesenchymal malignancy (9). Therefore, since radiological imaging findings are nonspecific in the preoperative diagnosis of IMT of renal pelvis, pathological examination combined with immunohistochemistry is essential to establish the final diagnosis of IMT of renal pelvis. It is usually difficult to differentiate between IMT of renal pelvis and other malignant tumors or conditions, such as blood clots of renal pelvis. The detection of ALK protein or rearrangements of the ALK gene has been previously considered a significant feature in the differential diagnosis of IMTs, but ALK ${ }^{+}$IMTs tend to occur more frequently in young patients, and their detection rate is low ( $\sim 65 \%$ of all IMTs) (10). In the present case, contrast-enhanced CT scan revealed the presence of a slightly enhanced tumor of $1.5 \times 1.0-\mathrm{cm}$ in size in the left renal pelvis, which mimicked renal pelvic carcinoma. Further examination on MRI was unable to differentiate between IMT, malignancies or blood clots of renal pelvis. Thus, the preoperative diagnosis of the patient was presumed to be renal pelvic carcinoma, according to his clinical symptoms and the radiological findings.

The preferred treatment for IMTs is radical resection of the tumor. In consequence, the patient was subjected to nephrectomy, based on the suspected diagnosis of renal pelvic carcinoma. However, examination of the intraoperative fast-frozen section did not identify any malignancy. IMTs of renal pelvis usually display a benign disease course, with no cases of relapse or metastasis reported in the literature (11). However, malignant progression of IMTs in other anatomic sites has previously been reported (12). IMTs usually present a good prognosis (5). In the present case, the patient has remained asymptomatic without any evidence of IMT for $>26$ months of follow-up.

In conclusion, IMTs of renal pelvis are rare tumors, which occasionally may cause iterative hematuria that is unresponsive to antibiotics. In previous cases reported in the literature, IMTs of renal pelvis were frequently misdiagnosed as malignancies or blood clots, and consequently, the patients were often subjected to nephrectomy or nephroureterectomy, based on a suspected diagnosis of renal pelvic carcinoma. Examination of an intraoperative fast-frozen section of the resected material may be aid in the treatment and differential diagnosis of IMT of renal pelvis, in order to avoid any unnecessary further ureterectomy (13).

In conclusion, the accurate diagnosis of IMTs remains a challenge. Therefore, the findings described in the present case report may contribute to improving the diagnostic accuracy of IMTs of renal pelvis.

\section{Acknowledgements}

The authors would like to thank the Departments of Radiology and Pathology of The Second Xiangya Hospital of Central South University (Changsha, China), for their support provided.

\section{References}

1. Coffin CM, Watterson J,PriestJR and DehnerLP: Extrapulmonary inflammatory myofibroblastic tumor (inflammatory pseudotumor). A clinicopathologic and immunohistochemical study of 84 cases. Am J Surg Pathol 19: 859-872, 1995.

2. Shah VB, Anchinmane VT and Savant D: Inflammatory myofibroblastic tumor of renal pelvis: A rare entity. Indian J Pathol Microbiol 54: 856-858, 2011.

3. Alquati S, Gira FA, Bartoli V, Contini S and Corradi D: Low-grade myofibroblastic proliferations of the urinary bladder. Arch Pathol Lab Med 137: 1117-1128, 2013.

4. Janik JS, Janik JP, Lovell MA, Hendrickson RJ, Bensard DD and Greffe BS: Recurrent inflammatory pseudotumors in children. J Pediatr Surg 38: 1491-1495, 2003.

5. Gleason BC and Hornick JL; Inflammatory myofibroblastic tumours: Where are we now? J Clin Pathol 61: 428-437, 2008.

6. Androulaki A, Papathomas TG, Liapis G, et al: Inflammatory pseudotumor associated with Mycobacterium tuberculosis infection. Int J Infect Dis 12: 607-610, 2008.

7. Li Z, Wang W, Wang Y, Zhai X, Tian Y, Fu Y and Zhou H: Inflammatory myofibroblastic tumor of the kidney with viral hepatitis B and trauma: A case report. Oncol Lett 6: 1741-1743, 2013.

8. Ho PH, Chen SY, Hsueh C, Lai MW, Chao HC and Chang PY: Inflammatory myofibroblastic tumor of renal pelvis presenting with prolonged fever and abdominal pain in children: Report of 1 case and review of literature. J Pediatr Surg 40: e35-e37, 2005.

9. Sedlic T, Scali EP, Lee WK, Verma S and Chang SD: Inflammatory pseudotumours in the abdomen and pelvis: A pictorial essay. Can Assoc Radiol J 65: 52-59, 2014.

10. Teoh JY, Chan NH, Cheung HY, Hou SS and Ng CF: Inflammatory myofibroblastic tumors of the urinary bladder: A systematic review. Urology 84: 503-508, 2014.

11. Kapusta LR, Weiss MA, Ramsay J, Lopez-Beltran A and Srigley JR: Inflammatory myofibroblastic tumors of the kidney: A clinicopathologic and immunohistochemical study of 12 cases. Am J Surg Pathol 27: 658-666, 2003.

12. Kim HW, Choi YH, Kang SM, Ku JY, Ahn JH, Kim JM, Chung JM, Ha HK and Chung MK: Malignant inflammatory myofibroblastic tumor of the bladder with rapid progression. Korean J Urol 53: 657-661, 2012.

13. Wu S, Chen L, Wan Q, Zhang L, Zhao X and Tang X: Inflammatory myofibroblastic tumor of the urinary bladder in a patient with the left renal cell carcinoma: A case report. Exp Ther Med 7: 1010-1012, 2014. 